\title{
Genetic Risk, Adherence to a Healthy Lifestyle, and Coronary Disease
}

\author{
Amit V. Khera, M.D., Connor A. Emdin, D.Phil., Isabel Drake, Ph.D., Pradeep Natarajan, \\ M.D., Alexander G. Bick, M.D., Ph.D., Nancy R. Cook, Ph.D., Daniel I. Chasman, Ph.D., \\ Usman Baber, M.D., Roxana Mehran, M.D., Daniel J. Rader, M.D., Valentin Fuster, M.D., \\ Ph.D., Eric Boerwinkle, Ph.D., Olle Melander, M.D., Ph.D., Marju Orho-Melander, Ph.D., Paul \\ M Ridker, M.D., and Sekar Kathiresan, M.D. \\ Center for Human Genetic Research and Cardiology Division, Massachusetts General Hospital \\ (A.V.K., P.N., S.K.), and the Division of Preventive Medicine, Department of Medicine, Brigham \\ and Women's Hospital (N.R.C., D.I.C., P.M.R.), Boston, and the Program in Medical and \\ Population Genetics, Broad Institute, Cambridge (A.V.K., C.A.E., A.G.B., S.K.) - all in \\ Massachusetts; the Department of Clinical Sciences, Lund University, Malmö, Sweden (I.D., O.M., \\ M.O.-M.); the Cardiovascular Institute, Mount Sinai Medical Center, Icahn School of Medicine at \\ Mount Sinai, New York (U.B., R.M., V.F.); Department of Genetics, Perelman School of Medicine \\ at the University of Pennsylvania, Philadelphia (D.J.R.); and the University of Texas Health \\ Science Center School of Public Health, Houston (E.B.)
}

\section{Abstract \\ BACKGROUND—Both genetic and lifestyle factors contribute to individual-level risk of coronary artery disease. The extent to which increased genetic risk can be offset by a healthy lifestyle is unknown.}

\begin{abstract}
METHODS—Using a polygenic score of DNA sequence polymorphisms, we quantified genetic risk for coronary artery disease in three prospective cohorts - 7814 participants in the Atherosclerosis Risk in Communities (ARIC) study, 21,222 in the Women's Genome Health Study (WGHS), and 22,389 in the Malmö Diet and Cancer Study (MDCS) - and in 4260 participants in the cross-sectional BioImage Study for whom genotype and covariate data were available. We also determined adherence to a healthy lifestyle among the participants using a scoring system consisting of four factors: no current smoking, no obesity, regular physical activity, and a healthy diet.
\end{abstract}

\begin{abstract}
RESULTS-The relative risk of incident coronary events was $91 \%$ higher among participants at high genetic risk (top quintile of polygenic scores) than among those at low genetic risk (bottom quintile of polygenic scores) (hazard ratio, 1.91; 95\% confidence interval [CI], 1.75 to 2.09). A favorable lifestyle (defined as at least three of the four healthy lifestyle factors) was associated
\end{abstract}

\footnotetext{
Address reprint requests to Dr. Kathiresan at the Center for Human Genetics Research, Massachusetts General Hospital, 185 Cambridge St., CPZN 5.252, Boston, MA 02114, or at skathiresan1@mgh.harvard.edu.

Drs. Khera and Emdin contributed equally to this article.

The views expressed in this article are those of the authors and do not necessarily represent the official views of Harvard Catalyst, Harvard University and its affiliated academic health care centers, or the National Institutes of Health.

Disclosure forms provided by the authors are available with the full text of this article at NEJM.org.
} 
with a substantially lower risk of coronary events than an unfavorable lifestyle (defined as no or only one healthy lifestyle factor), regardless of the genetic risk category. Among participants at high genetic risk, a favorable lifestyle was associated with a $46 \%$ lower relative risk of coronary events than an unfavorable lifestyle (hazard ratio, $0.54 ; 95 \% \mathrm{CI}, 0.47$ to 0.63 ). This finding corresponded to a reduction in the standardized 10-year incidence of coronary events from $10.7 \%$ for an unfavorable lifestyle to $5.1 \%$ for a favorable lifestyle in ARIC, from $4.6 \%$ to $2.0 \%$ in WGHS, and from $8.2 \%$ to $5.3 \%$ in MDCS. In the BioImage Study, a favorable lifestyle was associated with significantly less coronary-artery calcification within each genetic risk category.

CONCLUSIONS-Across four studies involving 55,685 participants, genetic and lifestyle factors were independently associated with susceptibility to coronary artery disease. Among participants at high genetic risk, a favorable lifestyle was associated with a nearly $50 \%$ lower relative risk of coronary artery disease than was an unfavorable lifestyle. (Funded by the National Institutes of Health and others.)

Both genetic and lifestyle factors are key drivers of coronary artery disease, a complex disorder that is the leading cause of death worldwide. ${ }^{1}$ A familial pattern in the risk of coronary artery disease was first described in 1938 and was subsequently confirmed in large studies involving twins and prospective cohorts. ${ }^{2-6}$ Since 2007, genomewide association analyses have identified more than 50 independent loci associated with the risk of coronary artery disease. ${ }^{7-15}$ These risk alleles, when aggregated into a polygenic risk score, are predictive of incident coronary events and provide a continuous and quantitative measure of genetic susceptibility. ${ }^{16-24}$

Much evidence has also shown that persons who adhere to a healthy lifestyle have markedly reduced rates of incident cardiovascular events. ${ }^{25-30}$ The promotion of healthy lifestyle behaviors, which include not smoking, avoiding obesity, regular physical activity, and a healthy diet pattern, underlies the current strategy to improve cardiovascular health in the general population. ${ }^{31}$

Many observers assume that a genetic predisposition to coronary artery disease is deterministic. ${ }^{32}$ However, genetic risk might be attenuated by a favorable lifestyle. Here, we analyzed data for participants in three prospective cohorts and one cross-sectional study to test the hypothesis that both genetic factors and baseline adherence to a healthy lifestyle contribute independently to the risk of incident coronary events and the prevalent subclinical burden of atherosclerosis. We then determined the extent to which a healthy lifestyle is associated with a reduced risk of coronary artery disease among participants with a high genetic risk.

\section{Methods}

\section{Study Populations}

The Atherosclerosis Risk in Communities (ARIC) study is a prospective cohort that enrolled white participants and black participants between the ages of 45 and 64 years, starting in 1987. ${ }^{33}$ For data from this study, we retrieved genotype and clinical data from the National Center for Biotechnology Information dbGAP server (accession number, phs000280.v3.p1). The Women's Genome Health Study (WGHS) is a prospective cohort of female health 
professionals derived from the Women's Health Study, a clinical trial initiated in 1992 to evaluate the efficacy of aspirin and vitamin $\mathrm{E}$ in the primary prevention of cardiovascular disease. ${ }^{34}$ The Malmö Diet and Cancer Study (MDCS) is a prospective cohort that enrolled participants between the ages of 44 and 73 years in Malmö, Sweden, starting in $1991 .{ }^{35} \mathrm{In}$ this study, participants with prevalent coronary disease at baseline were excluded. The BioImage Study enrolled asymptomatic participants between the ages of 55 and 80 years who were at risk for cardiovascular disease, beginning in 2008. This study included quantification of subclinical coronary artery disease in Agatston units, a metric that combines the area and density of observed coronary-artery calcification. ${ }^{36}$

\section{Polygenic Risk Score}

We derived a polygenic risk score from an analysis of up to 50 single-nucleotide polymorphisms (SNPs) that had achieved genomewide significance for association with coronary artery disease in previous studies. Details regarding the cohort-specific genotyping platform and risk scores are provided in Table S1 in the Supplementary Appendix, available with the full text of this article at NEJM.org. ${ }^{11-14}$ An example of the calculation of the polygenic risk score is provided in Table S2 in the Supplementary Appendix. Individual participant scores were created by adding up the number of risk alleles at each SNP and then multiplying the sum by the literature-based effect size. ${ }^{17}$ The genetic substructure of the population was assessed by calculating the principal components of ancestry. ${ }^{37}$

\section{Healthy Lifestyle Factors}

We adapted four healthy lifestyle factors from the strategic goals of the American Heart Association (AHA) - no current smoking, no obesity (body-mass index [the weight in kilograms divided by the square of the height in meters], <30), physical activity at least once weekly, and a healthy diet pattern. ${ }^{31} \mathrm{~A}$ healthy diet pattern was ascertained on the basis of adherence to at least half of the following recently endorsed characteristics ${ }^{38}$ : consumption of an increased amount of fruits, nuts, vegetables, whole grains, fish, and dairy products and a reduced amount of refined grains, processed meats, unprocessed red meats, sugarsweetened beverages, trans fats (WGHS only), and sodium (WGHS only). Because a detailed food-frequency questionnaire was not performed in the BioImage Study, diet scores in that cohort focused on self-reported consumption of fruits, vegetables, and fish. Additional details regarding cohort-specific metrics for lifestyle factors are provided in Table S3 in the Supplementary Appendix.

\section{Study End Points}

The primary study end point for the prospective cohort populations was a composite of coronary artery disease events that included myocardial infarction, coronary revascularization, and death from coronary causes. End-point adjudication was performed by a committee review of medical records within each cohort. In the BioImage Study, a crosssectional analysis of baseline scores for coronary-artery calcification was performed. 


\section{Statistical Analysis}

We used Cox proportional-hazard models to test the association of genetic and lifestyle factors with incident coronary events. We compared hazard ratios for participants at high genetic risk (i.e., highest quintile of polygenic scores) with those at intermediate risk (quintiles 2 to 4 ) or low risk (lowest quintile), as described previously. ${ }^{22,23}$ Similarly, we compared a favorable lifestyle (which was defined as the presence of at least three of the four healthy lifestyle factors) with an intermediate lifestyle (two healthy lifestyle factors) or an unfavorable lifestyle (no or only one healthy lifestyle factor). The primary analyses included adjustment for age, sex, self-reported education level, and the first five principal components of ancestry (unavailable in MDCS). In addition, WGHS analyses were adjusted for initial trial randomization to aspirin versus placebo and vitamin $\mathrm{E}$ versus placebo. We used Cox regression to calculate 10-year event rates, which were standardized to the mean of all predictor variables within each population. Because of a skewed distribution of scores for coronary-artery calcification in the BioImage Study, linear regression was performed on natural $\log$-transformed calcification scores with an offset of 1 . Predicted values were then reverse-transformed to calculate standardized scores, with higher values indicating an increased burden of coronary atherosclerosis. All the analyses were performed with the use of R software, version 3.1 (R Project for Statistical Computing).

\section{Results}

The populations in the prospective cohort studies included 7814 of 11,478 white participants in the ARIC cohort, 21,222 of 23,294 white women in the WGHS cohort, and 22,389 of 30,446 participants in the MDCS cohort for whom genotype and covariate data were available (Table 1). During follow-up, 1230 coronary events were observed in the ARIC cohort (median follow-up, 18.8 years), 971 coronary events in the WGHS cohort (median follow-up, 20.5 years), and 2902 coronary events in the MDCS cohort (median follow-up, 19.4 years) (Table S4 in the Supplementary Appendix). Categories of genetic and lifestyle risk were mutually independent within each cohort (Fig. S1 in the Supplementary Appendix).

Polygenic risk scores approximated a normal distribution within each cohort (Fig. S2 in the Supplementary Appendix). A risk gradient was noted across quintiles of genetic risk such that the participants at high genetic risk (i.e., in the top quintile of the polygenic scores) were at significantly higher risk of coronary events than those at low genetic risk (i.e., in the lowest quintile), with adjusted hazard ratios of 1.75 (95\% confidence interval [CI], 1.46 to 2.10) in the ARIC cohort, 1.94 (95\% CI, 1.58 to 2.39) in the WGHS cohort, and 1.98 (95\% CI, 1.76 to 2.23) in the MDCS cohort (Fig. 1, and Table S5 and Fig. S3 in the Supplementary Appendix). Across all three cohorts, the relative risk of incident coronary events was $91 \%$ higher among participants at high genetic risk than among those at low genetic risk (hazard ratio, $1.91 ; 95 \% \mathrm{CI}, 1.75$ to 2.09 ). A family history of coronary artery disease was an imperfect surrogate for genotype-defined risk, although the prevalence of such a self-reported family history tended to be higher among participants at high genetic risk than among those at low genetic risk. Levels of low-density lipoprotein (LDL) cholesterol were modestly increased across categories of genetic risk within each cohort. By 
contrast, genetic risk categories were independent of other cardiometabolic risk factors and 10-year cardiovascular risk as predicted by the pooled cohorts equation of the American College of Cardiology-AHA (Tables S6 through S9 in the Supplementary Appendix).

Each of the four healthy lifestyle factors was associated with a decreased risk of coronary events in a combined analysis of the prospective cohorts: no current smoking (hazard ratio, 0.56 ; $95 \% \mathrm{CI}, 0.47$ to 0.66 ), no obesity (hazard ratio, 0.66 ; $95 \% \mathrm{CI}, 0.58$ to 0.76 ), regular physical activity (hazard ratio, $0.88 ; 95 \% \mathrm{CI}, 0.80$ to 0.97 ), and healthy diet (hazard ratio, $0.91 ; 95 \%$ CI, 0.83 to 0.99 ) (Table S10 in the Supplementary Appendix). Coronary risk increased among participants with fewer healthy lifestyle factors within each cohort (Table S11 in the Supplementary Appendix).

Each cohort was divided into three lifestyle risk categories: favorable (at least three of the four healthy lifestyle factors), intermediate (two healthy lifestyle factors), or unfavorable (no or only one healthy lifestyle factor). Participants with an unfavorable lifestyle had higher rates of baseline hypertension and diabetes, a higher body-mass index, and less favorable levels of circulating lipids than did those with a favorable lifestyle (Tables S12, S13, and S14 in the Supplementary Appendix). An unfavorable lifestyle was associated with a higher risk of coronary events than a favorable lifestyle, with an adjusted hazard ratio of 1.71 (95\% CI, 1.47 to 1.98 ) in the ARIC cohort, 2.27 (95\% CI, 1.92 to 2.67) in the WGHS cohort, and 1.77 (95\% CI, 1.61 to 1.95) in the MDCS cohort (Fig. 1, and Fig. S3 in the Supplementary Appendix).

Within each category of genetic risk, lifestyle factors were strong predictors of coronary events (Fig. 2). Adherence to a favorable lifestyle, as compared with an unfavorable lifestyle, was associated with a $45 \%$ lower relative risk among participants at low genetic risk, a $47 \%$ lower relative risk among those at intermediate genetic risk, and a $46 \%$ lower relative risk (hazard ratio, $0.54 ; 95 \% \mathrm{CI}, 0.47$ to 0.63 ) among those at high genetic risk. Among participants at high genetic risk, the standardized 10-year coronary event rates were $10.7 \%$ among those with an unfavorable lifestyle and $5.1 \%$ among those with a favorable lifestyle in the ARIC cohort, $4.6 \%$ and $2.0 \%$, respectively, in the WGHS cohort, and $8.2 \%$ and $5.3 \%$ in the MDCS cohort (Fig. 3). Similarly, a low genetic risk was largely offset by an unfavorable lifestyle. Among participants at low genetic risk, standardized 10-year coronary event rates were 5.8\% among those with an unfavorable lifestyle and 3.1\% among those with a favorable lifestyle in the ARIC cohort, $1.8 \%$ and $1.2 \%$, respectively, in the WGHS cohort, and $4.7 \%$ and $2.6 \%$ in the MDCS cohort. Similar patterns were noted after the exclusion of coronary revascularization from the composite end point (Fig. S4 in the Supplementary Appendix). Adjustment for traditional risk factors attenuated estimates, although the decreased risk among participants with a favorable lifestyle within each genetic risk category remained apparent (Table S15 and Fig. S5 in the Supplementary Appendix).

Despite a paucity of well-validated genetic loci in black populations, we observed similar findings among black participants and white participants in the ARIC cohort (Fig. S6 in the Supplementary Appendix). However, additional data are needed to confirm the consistency of the effect in populations of African ancestry. 
A cross-sectional analysis of 4260 of 4301 white participants with available data from the BioImage Study showed that both genetic and lifestyle factors were associated with coronaryartery calcification (stratified according to the baseline characteristics in Tables S16 and S17 in the Supplementary Appendix). The standardized calcification score was 46 Agatston units (95\% CI, 39 to 54) among participants at high genetic risk, as compared with 21 Agatston units (95\% CI, 18 to 25$)$ among those at low genetic risk $(\mathrm{P}<0.001)$. The calcification score was similarly higher among participants with an unfavorable lifestyle than among those with a favorable lifestyle: 46 Agatston units (95\% CI, 40 to 53) versus 28 Agatston units (95\% CI, 25 to 31$)(\mathrm{P}<0.001)$. Within each subgroup of genetic risk, a significant trend was observed toward decreased coronaryartery calcification among participants who were more adherent to a healthy lifestyle (Fig. 4).

\section{Discussion}

In this study, we have provided quantitative data about the interplay between genetic and lifestyle risk factors for coronary artery disease in three prospective cohorts and one crosssectional study. High genetic risk was independent of healthy lifestyle behaviors and was associated with an increased risk (hazard ratio, 1.91) of coronary events and a substantially increased burden of coronary-artery calcification. However, within any genetic risk category, adherence to a healthy lifestyle was associated with a significantly decreased risk of both clinical coronary events and subclinical burden of coronary artery disease.

The results of this analysis support three noteworthy conclusions. First, our data indicate that inherited DNA variation and lifestyle factors contribute independently to a susceptibility to coronary artery disease. Our finding that a polygenic risk score has robust associations with incident coronary events is well aligned with previous studies of both primary and secondary prevention populations. ${ }^{16-24}$ Such findings support long-standing beliefs that genetic variants that are identifiable from birth alter coronary risk. ${ }^{2-4}$ Aside from slight differences in LDL cholesterol levels and a family history of coronary artery disease, genetic risk was independent of traditionally measured risk factors.

Second, a healthy lifestyle was associated with similar relative risk reductions in event rates across each stratum of genetic risk. Although the absolute risk reduction that was associated with adherence to a healthy lifestyle was greatest in the group at high genetic risk, our results support public health efforts that emphasize a healthy lifestyle for everyone. An alternative approach is to target intensive lifestyle modification to those at high genetic risk, with the expectation that disclosure of genetic risk can motivate behavioral change. However, whether the provision of such information can improve cardiovascular outcomes remains to be determined.

Third, patients may equate DNA-based risk estimates with determinism, a perceived lack of control over the ability to improve outcomes. ${ }^{32}$ However, our results provide evidence that lifestyle factors may powerfully modify risk regardless of the patient's genetic risk profile. Indeed, alternative analytic approaches that incorporate more stringent cutoffs or weight the relative effect for each healthy lifestyle factor may lead to an even more pronounced coronary risk gradient. 
Our study has several limitations. First, because adherence to a healthy lifestyle was not randomized, the association of lifestyle factors with the risk of coronary events cannot be taken as a causal relationship. Second, investigators in each cohort used slightly different methods to assess lifestyle at baseline. Behavioral changes before or after ascertainment or competing risks of other illnesses may have had an effect on risk estimates. Third, although we included up to 50 previously validated genetic polymorphisms in the polygenic risk score, the inclusion of even more variants may prove useful in future analyses. ${ }^{24}$ Finally, even though we provide evidence confirming a relationship between a polygenic risk score and coronary events among black participants, the generalizability of our findings should be tested in more diverse populations as more robust ethnicity-specific data regarding genetic association become available.

In conclusion, after quantifying both genetic and lifestyle risk among 55,685 participants in three prospective cohorts and one cross-sectional study, we found that adherence to a healthy lifestyle was associated with a substantially reduced risk of coronary artery disease within each category of genetic risk.

\section{Supplementary Material}

Refer to Web version on PubMed Central for supplementary material.

\section{Acknowledgments}

Supported by a grant from the American College of Cardiology- Merck Research Fellowship, a John S. Ladue Memorial Fellowship from Harvard Medical School, and a KL2/Catalyst Medical Research Investigator Training award from Harvard Catalyst funded by the National Institutes of Health (NIH) (TR001100, to Dr. Khera). Dr. Kathiresan is supported by an Ofer and Shelly Nemirovsky Research Scholar Award from Massachusetts General Hospital and grants from the NIH (HL127564 and UM1HG008895). Information on support for the cohort studies that are reviewed here is provided in the Supplementary Appendix.

We thank the investigators and participants in the ARIC, WGHS, MDCS, and BioImage studies for their contributions to this study.

\section{References}

1. Lozano R, Naghavi M, Foreman K, et al. Global and regional mortality from 235 causes of death for 20 age groups in 1990 and 2010: a systematic analysis for the Global Burden of Disease Study 2010. Lancet. 2012; 380:2095-2128. [PubMed: 23245604]

2. Müller C. Xanthomata, hypercholesterolemia, angina pectoris. Acta Med Scand. 1938; 89:75-84.

3. Gertler MM, Garn SM, White PD. Young candidates for coronary heart disease. J Am Med Assoc. 1951; 147:621-625. [PubMed: 14873513]

4. Slack J, Evans KA. The increased risk of death from ischaemic heart disease in first degree relatives of 121 men and 96 women with ischaemic heart disease. J Med Genet. 1966; 3:239-257. [PubMed: 16175706]

5. Marenberg ME, Risch N, Berkman LF, Floderus B, de Faire U. Genetic susceptibility to death from coronary heart disease in a study of twins. N Engl J Med. 1994; 330:1041-1046. [PubMed: 8127331]

6. Lloyd-Jones DM, Nam BH, D'Agostino RB Sr, et al. Parental cardiovascular disease as a risk factor for cardiovascular disease in middle-aged adults: a prospective study of parents and offspring. JAMA. 2004; 291:2204-2211. [PubMed: 15138242]

7. Samani NJ, Erdmann J, Hall AS, et al. Genomewide association analysis of coronary artery disease. N Engl J Med. 2007; 357:443-453. [PubMed: 17634449] 
8. Helgadottir A, Thorleifsson G, Manolescu A, et al. A common variant on chromosome 9p21 affects the risk of myocardial infarction. Science. 2007; 316:1491-1493. [PubMed: 17478679]

9. McPherson R, Pertsemlidis A, Kavaslar N, et al. A common allele on chromosome 9 associated with coronary heart disease. Science. 2007; 316:1488-1491. [PubMed: 17478681]

10. Myocardial Infarction Genetics Consortium. Genome-wide association of early-onset myocardial infarction with single nucleotide polymorphisms and copy number variants. Nat Genet. 2009; 41:334-341. [PubMed: 19198609]

11. Erdmann J, Grosshennig A, Braund PS, et al. New susceptibility locus for coronary artery disease on chromosome 3q22.3. Nat Genet. 2009; 41:280-282. [PubMed: 19198612]

12. Coronary Artery Disease (C4D) Genetics Consortium. A genome-wide association study in Europeans and South Asians identifies five new loci for coronary artery disease. Nat Genet. 2011; 43:339-344. [PubMed: 21378988]

13. IBC 50K CAD Consortium. Large-scale gene-centric analysis identifies novel variants for coronary artery disease. PLoS Genet. 2011; 7(9):e1002260. [PubMed: 21966275]

14. The CARDIoGRAMplusC4D Consortium. Large-scale association analysis identifies new risk loci for coronary artery disease. Nat Genet. 2013; 45:25-33. [PubMed: 23202125]

15. Nikpay M, Goel A, Won HH, et al. A comprehensive 1,000 Genomes-based genome-wide association meta-analysis of coronary artery disease. Nat Genet. 2015; 47:1121-1130. [PubMed: 26343387]

16. Kathiresan S, Melander O, Anevski D, et al. Polymorphisms associated with cholesterol and risk of cardiovascular events. N Engl J Med. 2008; 358:1240-1249. [PubMed: 18354102]

17. Ripatti S, Tikkanen E, Orho-Melander M, et al. A multilocus genetic risk score for coronary heart disease: case-control and prospective cohort analyses. Lancet. 2010; 376:1393-1400. [PubMed: 20971364]

18. Paynter NP, Chasman DI, Paré G, et al. Association between a literature-based genetic risk score and cardiovascular events in women. JAMA. 2010; 303:631-637. [PubMed: 20159871]

19. Thanassoulis G, Peloso GM, Pencina MJ, et al. A genetic risk score is associated with incident cardiovascular disease and coronary artery calcium: the Framingham Heart Study. Circ Cardiovasc Genet. 2012; 5:113-121. [PubMed: 22235037]

20. Brautbar A, Pompeii LA, Dehghan A, et al. A genetic risk score based on direct associations with coronary heart disease improves coronary heart disease risk prediction in the Atherosclerosis Risk in Communities (ARIC), but not in the Rotterdam and Framingham Offspring, Studies. Atherosclerosis. 2012; 223:421-426. [PubMed: 22789513]

21. Ganna A, Magnusson PK, Pedersen NL, et al. Multilocus genetic risk scores for coronary heart disease prediction. Arterioscler Thromb Vasc Biol. 2013; 33:2267-2272. [PubMed: 23685553]

22. Mega JL, Stitziel NO, Smith JG, et al. Genetic risk, coronary heart disease events, and the clinical benefit of statin therapy: an analysis of primary and secondary prevention trials. Lancet. 2015; 385:2264-2271. [PubMed: 25748612]

23. Tada H, Melander O, Louie JZ, et al. Risk prediction by genetic risk scores for coronary heart disease is independent of self-reported family history. Eur Heart J. 2016; 37:561-567. [PubMed: 26392438]

24. Abraham G, Havulinna AS, Bhalala OG, et al. Genomic prediction of coronary heart disease. Eur Heart J. 2016 Sep 21. Epub ahead of print.

25. Stampfer MJ, Hu FB, Manson JE, Rimm EB, Willett WC. Primary prevention of coronary heart disease in women through diet and lifestyle. N Engl J Med. 2000; 343:16-22. [PubMed: 10882764]

26. Folsom AR, Yatsuya H, Nettleton JA, Lutsey PL, Cushman M, Rosamond WD. Community prevalence of ideal cardiovascular health, by the American Heart Association definition, and relationship with cardiovascular disease incidence. J Am Coll Cardiol. 2011; 57:1690-1696. [PubMed: 21492767]

27. Yang Q, Cogswell ME, Flanders WD, et al. Trends in cardiovascular health metrics and associations with all-cause and CVD mortality among US adults. JAMA. 2012; 307:1273-1283. [PubMed: 22427615] 
28. Xanthakis V, Enserro DM, Murabito JM, et al. Ideal cardiovascular health: associations with biomarkers and subclinical disease and impact on incidence of cardiovascular disease in the Framingham Offspring Study. Circulation. 2014; 130:1676-1683. [PubMed: 25274000]

29. Chomistek AK, Chiuve SE, Eliassen AH, Mukamal KJ, Willett WC, Rimm EB. Healthy lifestyle in the primordial prevention of cardiovascular disease among young women. J Am Coll Cardiol. 2015; 65:43-51. [PubMed: 25572509]

30. Akesson A, Larsson SC, Discacciati A, Wolk A. Low-risk diet and lifestyle habits in the primary prevention of myocardial infarction in men: a population-based prospective cohort study. $\mathrm{J}$ Am Coll Cardiol. 2014; 64:1299-1306. [PubMed: 25257629]

31. Lloyd-Jones DM, Hong Y, Labarthe D, et al. Defining and setting national goals for cardiovascular health promotion and disease reduction: the American Heart Association's strategic Impact Goal through 2020 and beyond. Circulation. 2010; 121:586-613. [PubMed: 20089546]

32. White PD. Genes, the heart and destiny. N Engl J Med. 1957; 256:965-969. [PubMed: 13451977]

33. The Atherosclerosis Risk in Communities (ARIC) Study: design and objectives. Am J Epidemiol. 1989; 129:687-702. [PubMed: 2646917]

34. Ridker PM, Chasman DI, Zee RY, et al. Rationale, design, and methodology of the Women's Genome Health Study: a genome-wide association study of more than 25,000 initially healthy American women. Clin Chem. 2008; 54:249-255. [PubMed: 18070814]

35. Berglund G, Elmstähl S, Janzon L, Larsson SA. The Malmo Diet and Cancer Study: design and feasibility. J Intern Med. 1993; 233:45-51. [PubMed: 8429286]

36. Baber U, Mehran R, Sartori S, et al. Prevalence, impact, and predictive value of detecting subclinical coronary and carotid atherosclerosis in asymptomatic adults: the BioImage study. J Am Coll Cardiol. 2015; 65:1065-1074. [PubMed: 25790876]

37. Price AL, Patterson NJ, Plenge RM, Weinblatt ME, Shadick NA, Reich D. Principal components analysis corrects for stratification in genome-wide association studies. Nat Genet. 2006; 38:904 909. [PubMed: 16862161]

38. Mozaffarian D. Dietary and policy priorities for cardiovascular disease, diabetes, and obesity: a comprehensive review. Circulation. 2016; 133:187-225. [PubMed: 26746178] 

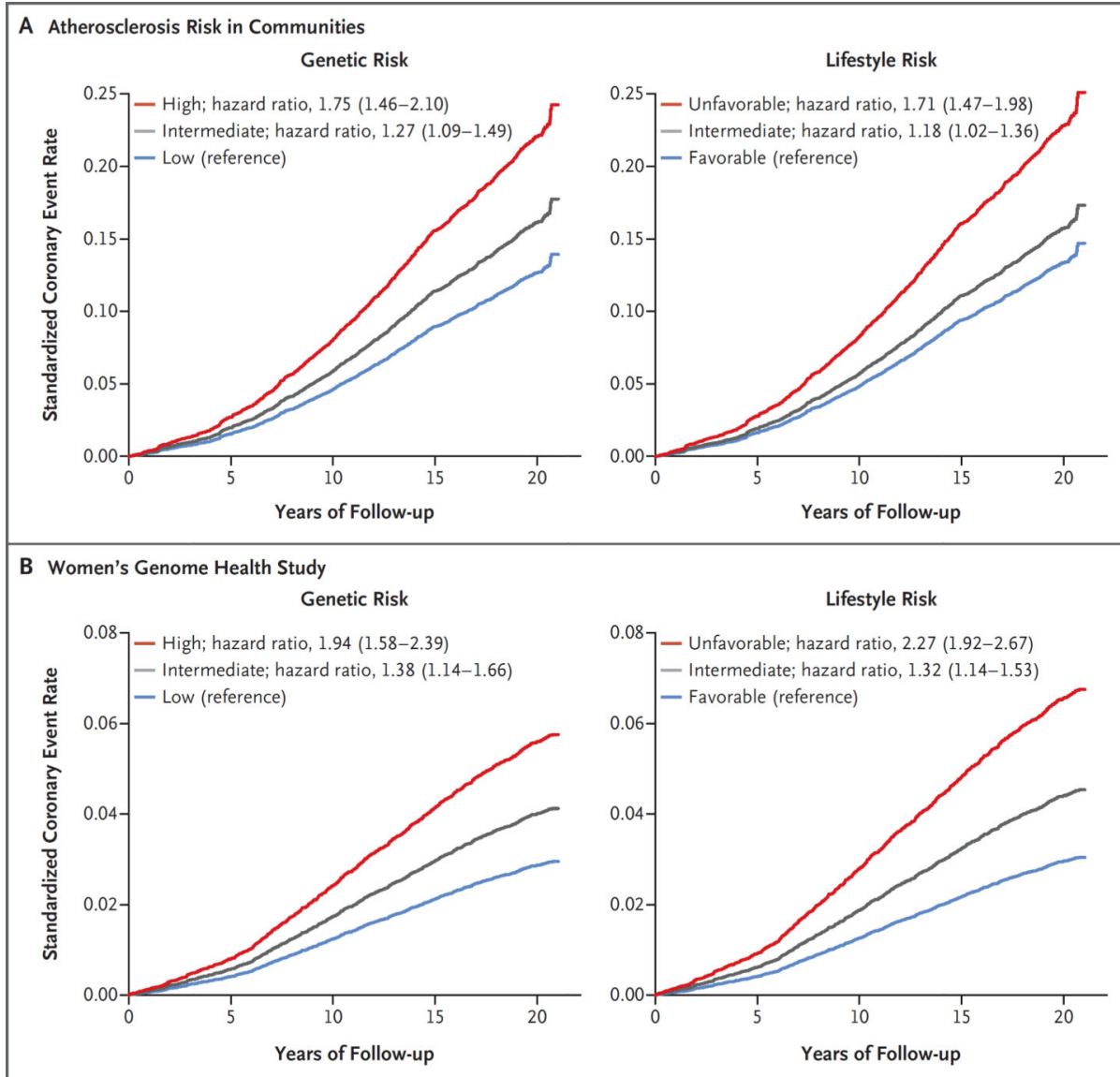

C Malmö Diet and Cancer Study
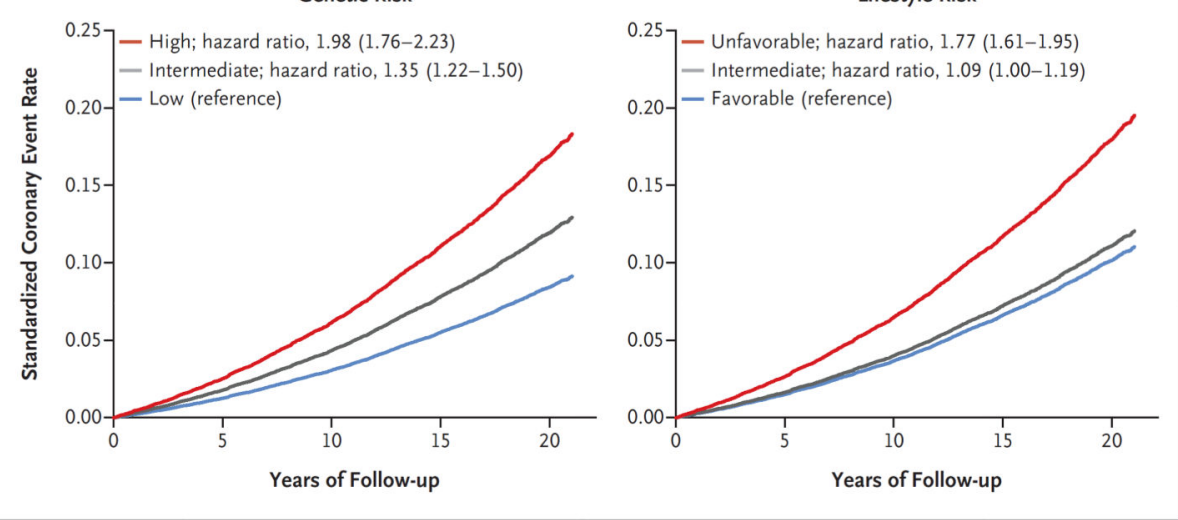

Figure 1. Standardized Coronary Events Rates, According to Genetic and Lifestyle Risk in the Prospective Cohorts

Shown are the standardized rates of coronary events, according to the genetic risk and lifestyle risk of participants in the Atherosclerosis Risk in Communities (ARIC) cohort, the Women's Genome Health Study (WGHS) cohort, and the Malmö Diet and Cancer Study (MDCS) cohort. The $95 \%$ confidence intervals for the hazard ratios are provided in parentheses. Cox regression models were adjusted for age, sex (in ARIC and MDCS), randomization to receive vitamin $\mathrm{E}$ or aspirin (in WGHS), education level, and principal 
components of ancestry (in ARIC and WGHS). Standardization was performed to cohortspecific population averages for each covariate. 


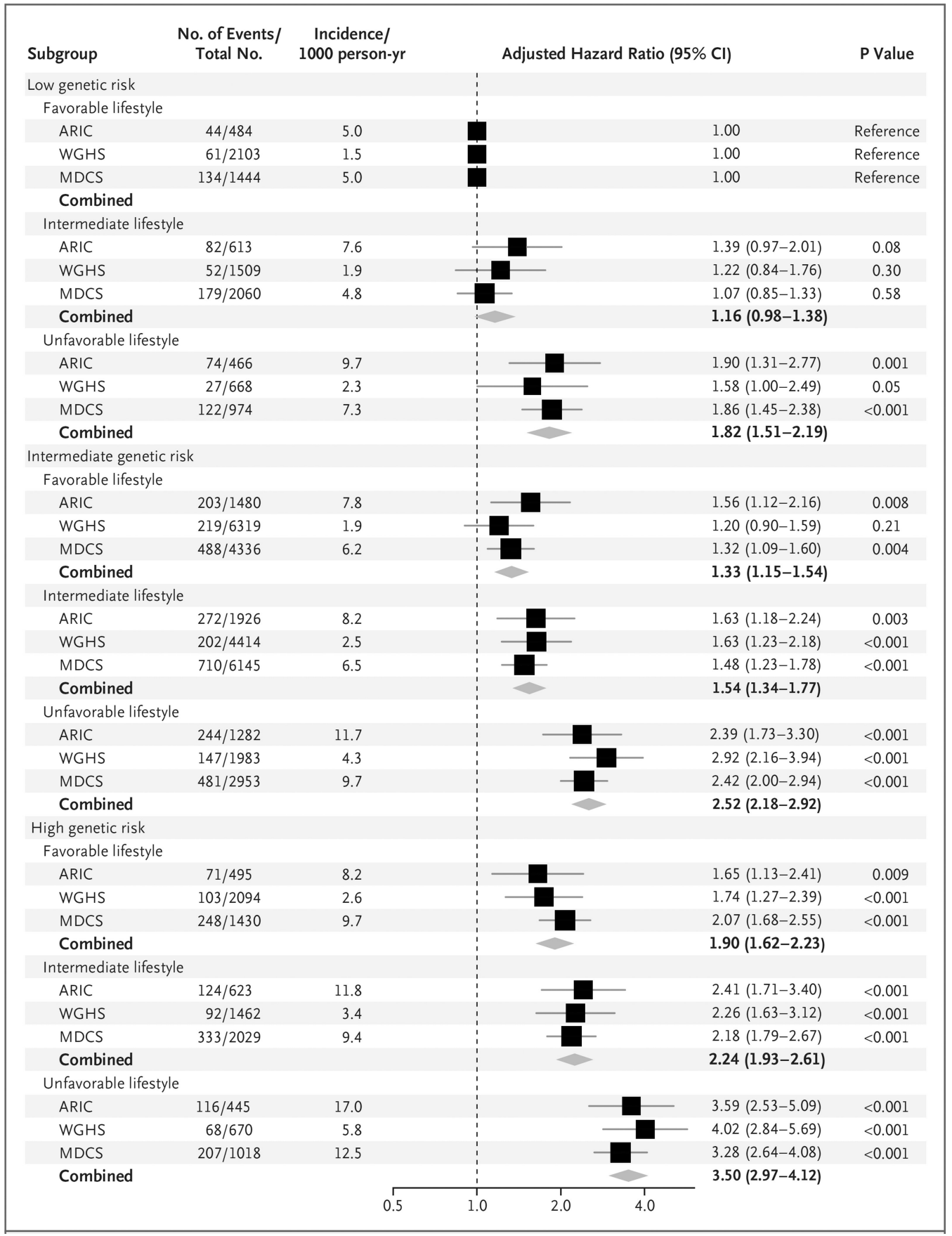

Figure 2. Risk of Coronary Events, According to Genetic and Lifestyle Risk in the Prospective Cohorts

Shown are adjusted hazard ratios for coronary events in each of the three prospective cohorts, according to genetic risk and lifestyle risk. In these comparisons, participants at low genetic risk with a favorable lifestyle served as the reference group. There was no evidence of a significant interaction between genetic and lifestyle risk factors $(\mathrm{P}=0.38$ for interaction in the Atherosclerosis Risk in Communities (ARIC) cohort, $\mathrm{P}=0.31$ in the Women's Genome Health Study (WGHS) cohort, and P = 0.24 in the Malmö Diet and Cancer Study 
(MDCS) cohort). Unadjusted incidence rates are reported per 1000 person-years of followup. A random-effects meta-analysis was used to combine cohort-specific results. 
Favorable lifestyle

Intermediate lifestyle

Unfavorable lifestyle
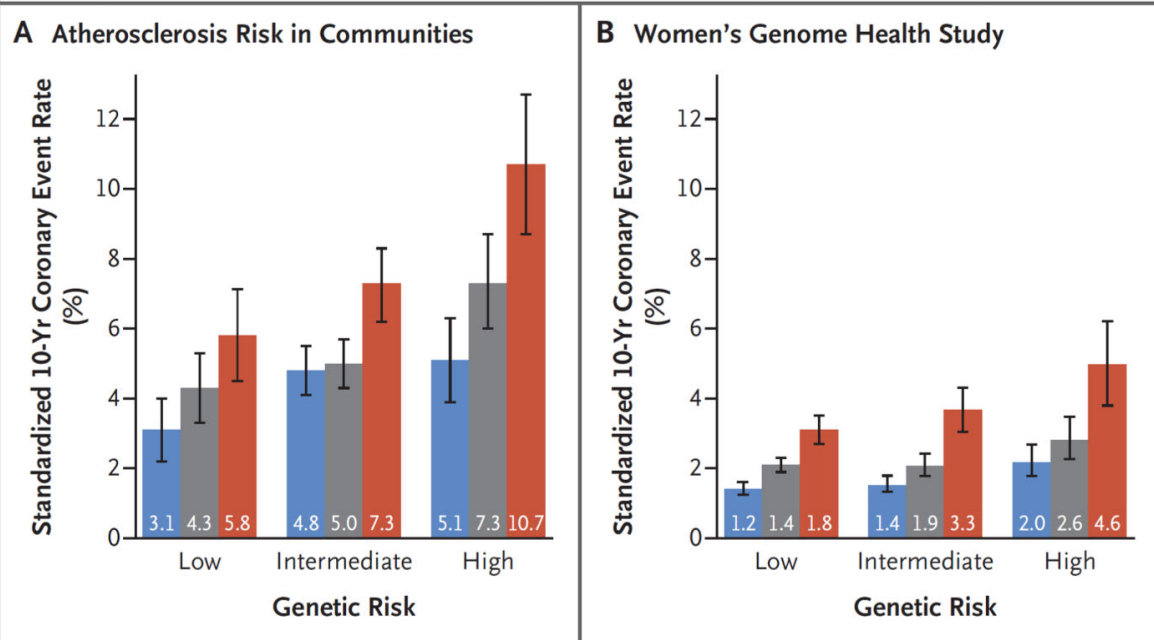

C Malmö Diet and Cancer Study

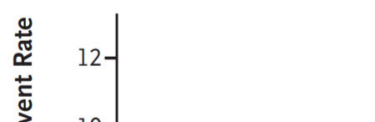

Figure 3. 10-Year Coronary Event Rates, According to Lifestyle and Genetic Risk in the Prospective Cohorts

Shown are standardized 10-year cumulative incidence rates for coronary events in the three prospective cohorts, according to lifestyle and genetic risk. Standardization was performed to cohort-specific population averages for each covariate. The I bars represent $95 \%$ confidence intervals. 

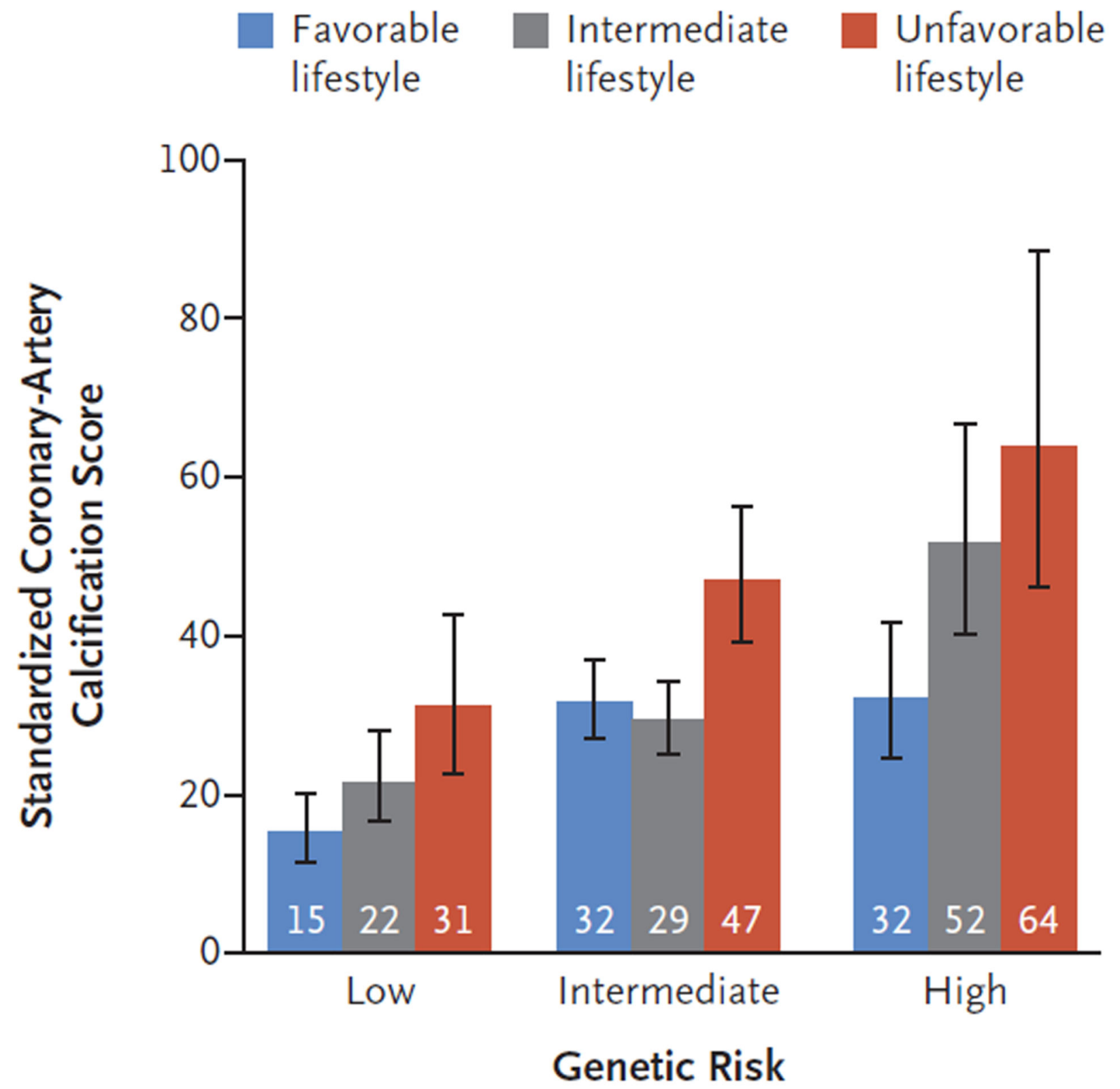

Figure 4. Coronary-Artery Calcification Score in the BioImage Study, According to Lifestyle and Genetic Risk

Among the participants in the BioImage Study, a standardized score for coronary-artery calcification was determined by means of linear regression after adjustment for age, sex, education level, and principal components of ancestry. Standardization was performed on the basis of study averages for each covariate. Average standardized coronary-artery calcification scores are expressed in Agatston units, with higher scores indicating an increased burden of coronary atherosclerosis. The I bars represent $95 \%$ confidence intervals. 


\section{Table 1}

Characteristics of the Participants at Baseline. ${ }^{*}$

\begin{tabular}{|c|c|c|c|c|}
\hline Characteristic & $\begin{array}{l}\text { Atherosclerosis Risk } \\
\text { in Communities } \\
(\mathbf{N}=\mathbf{7 8 1 4})\end{array}$ & $\begin{array}{c}\text { Women's Genome } \\
\text { Health Study } \\
(\mathbf{N}=\mathbf{2 1 , 2 2 2})\end{array}$ & $\begin{array}{c}\text { Malmö Diet and } \\
\text { Cancer Study } \\
(\mathbf{N}=\mathbf{2 2 , 3 8 9 )}\end{array}$ & $\begin{array}{c}\text { BioImage Study } \\
\quad(\mathrm{N}=\mathbf{4 2 6 0})\end{array}$ \\
\hline Age - yr & $54 \pm 5.7$ & $54.2 \pm 7.1$ & $58.0 \pm 7.7$ & $69.1 \pm 6.0$ \\
\hline Male sex - no. (\%) & $3555(45)$ & 0 & $8,515(38)$ & $1879(44)$ \\
\hline \multicolumn{5}{|l|}{ Clinical history — no./total no. (\%) } \\
\hline Hypertension & 2020/7784 (26) & $5164 / 21,217(24)$ & $13,553 / 22,389(61)$ & $2576 / 4258(60)$ \\
\hline Diabetes mellitus & $632 / 7799(8)$ & $519 / 21,222(2)$ & $904 / 22,389(4)$ & $522 / 4257(12)$ \\
\hline $\begin{array}{l}\text { Family history of premature coro- } \\
\text { nary artery disease }{ }^{\dagger}\end{array}$ & $751 / 6812(11)$ & $2476 / 19,121(13)$ & $7,225 / 22,389(32)$ & $1717 / 4054(42)$ \\
\hline Body-mass index ${ }^{t}$ & $26.9 \pm 4.8$ & $25.9 \pm 4.9$ & $25.7 \pm 3.9$ & $28.8 \pm 5.5$ \\
\hline \multicolumn{5}{|l|}{ Lipid levels $-\mathrm{mg} / \mathrm{dl} \xi$} \\
\hline LDL cholesterol & $136.7 \pm 38.7$ & $124 \pm 34$ & $161.2 \pm 38.6$ & $113 \pm 33$ \\
\hline HDL cholesterol & $37.6 \pm 10.9$ & $54 \pm 15$ & $53.7 \pm 14.7$ & $56 \pm 16$ \\
\hline Median triglycerides (IQR) & $110(79-156)$ & $119(84-176)$ & $102(76-143)$ & $148(107-210)$ \\
\hline $\begin{array}{l}\text { Use of lipid-lowering medication } \\
- \text { no. }(\%)\end{array}$ & $45(1)$ & $690(3)$ & $488(2)$ & $1467(34)$ \\
\hline \multicolumn{5}{|l|}{ Healthy lifestyle factors - no. (\%) } \\
\hline No current smoking & $5873(75)$ & $18,784(89)$ & $16,162(72)$ & $3887(91)$ \\
\hline No obesity & $6093(78)$ & $17,566(83)$ & $19,507(87)$ & $2729(64)$ \\
\hline Regular physical activity & $2743(35)$ & $9,256(44)$ & $9,093(41)$ & $1967(46)$ \\
\hline Healthy diet & $1515(19)$ & $7,251(34)$ & $2,795(12)$ & $610(14)$ \\
\hline \multicolumn{5}{|l|}{ Healthy lifestyle score - no. (\%) } \\
\hline 3 or 4 healthy lifestyle factors & $2459(31)$ & $10,516(50)$ & $7,210(32)$ & $1564(37)$ \\
\hline 2 healthy lifestyle factors & $3162(40)$ & $7,385(35)$ & $10,234(46)$ & $1598(38)$ \\
\hline 0 or 1 healthy lifestyle factor & $2193(28)$ & $3,321(16)$ & $4,945(22)$ & $1098(26)$ \\
\hline \multicolumn{5}{|l|}{ Genetic risk category - no. $(\%)$} \\
\hline Low & $1563(20)$ & $4,280(20)$ & $4,478(20)$ & $846(20)$ \\
\hline Intermediate & $4688(60)$ & $12,716(60)$ & $13,434(60)$ & $2557(60)$ \\
\hline High & $1563(20)$ & $4,226(20)$ & $4,477(20)$ & $857(20)$ \\
\hline
\end{tabular}

Plus-minus values are means \pm SD. P values for the differences between the study groups in each individual cohort at baseline are provided in the tables in the Supplementary Appendix. To convert the values for cholesterol to millimoles per liter, multiply by 0.02586 . To convert the values for triglycerides to millimoles per liter, multiply by 0.01129 . HDL denotes high-density lipoprotein, IQR interquartile range, and LDL low-density lipoprotein.

\footnotetext{
${ }^{\dagger}$ A family history of premature coronary artery disease refers to a self-reported parental history of myocardial infarction before the age of 60 years. In the BioImage Study and the Malmö Diet and Cancer Study (MDCS), participants were asked about a parental history of myocardial infarction without an age restriction.

${ }^{*}$ The body-mass index is the weight in kilograms divided by the square of the height in meters.

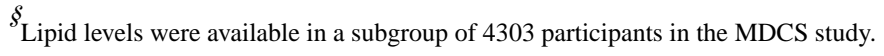

